\title{
A Multi-Functional De-Icing Equipment Using Modular Parallel PWM Current Source Converters
}

\author{
Yang Hu ${ }^{1}$, Heng Zhang ${ }^{1}$, Jing Chen ${ }^{1}$, Huan Zhang ${ }^{1}$, Xinmeng Liu ${ }^{1}$, Jiachen $\mathrm{Li}^{1}{ }^{1}$, Hanyu Chen ${ }^{1}$, Haiyang Hu ${ }^{1}$ \\ ${ }^{1}$ State Grid Corporation of China, China
}

\begin{abstract}
For transmission line de-icing, a five level specific harmonic elimination (51-she) modulation method for high-power parallel current source is proposed, which realizes the current balance of DC bridge arm and the suppression of common mode voltage, and ensures the power quality of grid connected current at low switching frequency. In addition the reactive power of the low voltage load can be compensated at the same time. Firstly, the switching states are classified according to the different mode lengths of PWM current, and the common mode voltage corresponding to each switching state is calculated. Secondly, the current sharing control strategy is established based on the analysis of the influence of redundant switching state on the current sharing of DC bridge arm. Then, the 51-she waveform is constructed based on the switching state with lower common mode voltage, and the redundant switching states is optimized according to the current sharing strategy. Finally, the effectiveness of the proposed method is verified by simulation.
\end{abstract}

\section{Introduction}

Heavy icing on transmission lines can cause serious damages to modern power system equipment such as the line broken and the collapses of tower, which may even lead to further serious consequences including power system blackout. These accidents have been reported in recent years [1]-[3].

To ensure the secure operation of transmission lines and towers, active de-icing through using power electronics equipment has gained growing interest in recent years. There are two types of de-icing approaches, namely ac de-icing and dc de-icing, according to the type of the current flowing through the transmission line. When ac current is applied, it is nontrivial amount of reactive power flow on the line, which is a huge loss regarding power converter capacity. Alternatively, dc deicing has been considered as a good candidate due to its simple control and there is no involvement of reactive power flow.

In the previous study, the thyristor based line commutated converter has been adopted to provide high dc current. However, this method is associated with high dc current ripples and the ac current has significant harmonic distortions which need to be compensated by using either passive or active harmonic filter [4].

On the other hand, in recent years, the modular multilevel voltage source converter has been considered as an alternative candidate. Comparing to the line commutated converters, a PWM voltage source can accurate control the ac line current with minimized distortion [5]. However, as VSC based rectifier only controls the dc voltage instead of the dc current, the accuracy of de-icing current regulation can be affected. In more recent years, the modular multi-level converter has been studied to dc de-icing application. Comparing to the conventional twolevel voltage source converter, MMC system features modular configuration that is easy for maintenance and capacity expansion. In addition, the possibility of using de-icing equipment for auxiliary services including reactive power compensation has been discussed. However, it is necessary to point out that MMC system is typically bulky and cost expensive and the control of MMC is complicated.

Moreover, it is seen that the PWM current source converter (CSC) has been widely used in high power applications including medium voltage variable speed drives and energy storage using superconductive coils. As the dc current is fully controlled, it is considered as a good candidate for dc de-icing. However, the capacity of a single CSC is limited due to the limitation of withstand voltage and current of power electronics devices. To enhance to power transfer capacity, using parallel CSC modules to construct a high power system is study. In [6], a direct parallel connection of CSC modules is proposed and the selection of space vectors is discussed in order to obtain proper dc rail current balancing.

To providing high amplitude dc high current, ac-dc rectifiers are needed. Firstly, the thyristor based line commutated converter (LCC) can be used to provide high de current. However, LCC converter is associated with the possibility of communicate failure and it can produce significant harmonics when the output $\mathrm{dc}$ voltage is tuned in a wide range. Alternatively, voltage source converter (VSC) based equipment has been

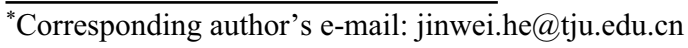


developed in recent years. Comparing to LCC counterparts, the traditional VSC has better harmonic control performance and the output voltage can be accurately tuned. However, for a single two-level VSC converter, it is difficult to reach the required high dc voltage for de-icing unless the high voltage power switching device is used. This certainly associated with high cost and the power loss. In addition, conventional VSC is only used for de-icing, it is a fact that the flexibility and controllability have not been well exploited yet.

To deal with the aforementioned limitation, the multilevel VSC, including modular multi-level converter (MMC), the cascaded H-bridge converter ( $\mathrm{CHB})$, and the combination of them, have been applied to the dc deicing. The major merits of these converters are scalability, the inherent feature of redundancy, and the superior harmonic performance in the ac sider of the converter. In addition, when the converter works in idle mode or only a portion of the capacity is dispatched for dc de-icing, they can also be used to other purposes in including the static var compensation and the local harmonic mitigation. For instance, a MMC converter based dc deicing method has been proposed, where it is used to provide auxiliary reactive power compensation and the cooperative operation with de-icing is discussed.

It is necessary to note that the above-mentioned method is based on VSCs. Conventionally, the VSC based rectifier only controls dc rail voltage instead of dc current. While regarding the de-icing, it is preferred to have a direct regulation of coil current. Alternatively, it is found that the PWM current source converter (CSC) has been received growing attentions in recent years. Comparing to VSCs, a CSC can realize a direct closedloop control of dc current with simple control complexity. In addition, it can easily block the short circuit faults which are typically challenging of VSCs counterparts.

When applying CSC to dc de-icing application, an important task is to provide high de current at low cost and high reliability. To meet with these requirements, in this paper, a parallel modular CSC based dc de-icing converter is proposed. Firstly, the proposed system adopts a closed-loop control of dc rail voltage for deicing. Then, in order to exploit the capability of the parallel CSCs, the local load reactive power compensation is included in the CSCs controller. Finally, in order to provide the high dc current for various application, the current balancing between parallel CSC modules is discussed. It has been investigated that both the small and medium current vector can be used for dc current balancing. Considering the CSC is usually operated in low modulation index region for providing high dc current, only the small vectors are used for current balancing. Experimental results have been provided to validate the correctness of the proposed dc de-icing approach.

\section{The proposed system}

This paper starts with the introduction to the configuration of the proposed system. Afterwards, the detailed control principle is provided.

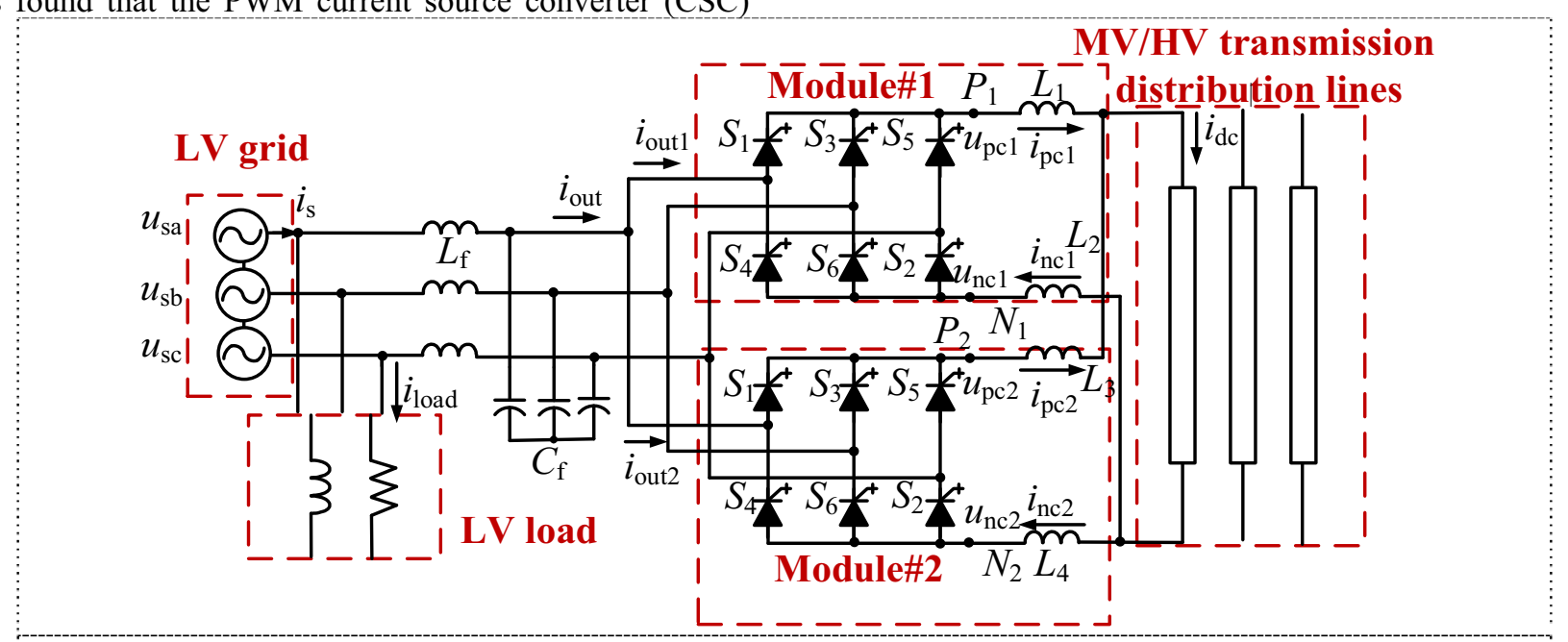

Figure 1. Circuit diagram of the proposed parallel current source converter based dc de-icing system.

\subsection{Circuit configuration}

Fig. 1 shows the circuit configurations of the proposed system, where two parallel PWM CSC modules are connected to the low voltage three-phase grid with a CL filter. For the CSC converters, each module has six devices as $S_{1}$ to $S_{6} . u_{\mathrm{sa}}, u_{\mathrm{sb}}$, and $u_{\mathrm{sc}}$ are the three-phase grid voltage, $C_{\mathrm{f}}$ is the capacitance of the CL filter, and $L_{\mathrm{f}}$ is the inductance of the CL filter, $i_{\mathrm{s}}$ is the line current after CL filtering, $i_{\text {out }}$ is the output current of the CSC, and $i_{\text {load }}$ is local load current.

In the right-hand side of the CSC, each module has its upper and lower dc rail as $P_{1}, N_{1}, P_{2}$, and $N_{2}$. The current flows through the dc rails are $i_{\mathrm{pc} 1}, i_{\mathrm{nc} 1}, i_{\mathrm{pc} 2}$ and $i_{\mathrm{nc} 2}$. Afterwards, the sum of the dc current $i_{\mathrm{dc}}$ is applied to the transmission line for dc-icing. In order to de-icing the three phase line in a proper sequence, there are a few dc disconnectors at the dc terminal of the CSCs.

For this system, the primary task is to provide high dc current for de-icing. In addition, it also has the capability of compensating local load reactive power through 
injecting an opposite current. According to this, the system is composed of three modes:

(1) De-icing mode: In this case, the CSC generates a stable high dc current that feeds to the transmission line. At the same time, the line current is in phase with the three phase grid voltage.

(2) Reactive power compensation mode: In this operation mode, line current is opposite to the reactive power current from the local load. Meanwhile, in order to reduce the power loss on the dc chokes, the dc current actively is tuned to be slightly higher that the peak of line current.

(3) Multi-functional mode: In this operation mode, both the requirement of dc-icing and the reactive power compensation shall be satisfied.

\subsection{Controller design}

To meet with the abovementioned tasks, the controller of the system shall be carefully designed. Firstly, considering that the primary task for this system is deicing, the dc current control loop is developed as given in:

$$
\begin{gathered}
I_{\mathrm{d}, \mathrm{ref}}=\left(k_{\mathrm{p}, \mathrm{dc}}+\frac{k_{\mathrm{i}, \mathrm{dc}}}{s}\right) \cdot\left(i_{\mathrm{dc}, \mathrm{ref}}-i_{\mathrm{dc}, \mathrm{LPF}}\right) \\
i_{\mathrm{dc}, \mathrm{LPF}}=\frac{\tau}{s+\tau} i_{\mathrm{dc}}
\end{gathered}
$$

where $i_{\mathrm{dc}}$ is measured dc current, $i_{\mathrm{dc}, \text { LPF }}$ is the measured dc current after a low-pass filtering, $i_{\mathrm{dc}}$, ref is the reference current for de-icing, $k_{\mathrm{p}, \mathrm{dc}}$ is the proportional gain of the dc current regulator and $k_{\mathrm{i}, \mathrm{dc}}$ is the integral gain of the regulator. The output of the regulator $I_{\mathrm{d} \text {, ref }}$ is the $d$ axis reference current for modulation.

For low voltage local load reactive power compensation, it is necessary to extract the reactive power at first as:

$$
Q_{\text {load }}=\frac{3}{2}\left(v_{\mathrm{g}, \alpha} \cdot i_{\text {load }, \beta}-v_{\mathrm{g}, \beta} \cdot i_{\text {load }, \alpha}\right)
$$

where $Q_{\text {load }}$ is the measured reactive power, $v_{\mathrm{g}, \alpha}$ and $v_{\mathrm{g}, \beta}$ are the instant grid voltage in the two-axis stationary reference frame, and $i_{\text {load, } \alpha}$ and $i_{\text {load, } \beta}$ are the load current in the two-axis stationary reference frame.

Then, the reactive power current component is determined and it can be used as the reference current of the CSC as:

$$
\begin{gathered}
I_{\mathrm{q}, \text { load }}=\frac{2 Q_{\mathrm{Load}}}{3 E_{\mathrm{g}}} \\
I_{\mathrm{q}, \text { ref }}=\frac{2 Q_{\mathrm{Load}}}{3 E_{\mathrm{g}}}
\end{gathered}
$$

When both the real power current component and reactive power component are determined, the modulation index $m_{\text {ref }}$ and the angle of reference current $\theta_{\text {ref }}$ can be easily determined as:

$$
\begin{gathered}
\theta_{\text {ref }}=\operatorname{Tan}^{-1}\left(\frac{I_{\mathrm{q}, \text { ref }}}{I_{\mathrm{d}, \text { ref }}}\right)+\theta_{\text {grid }} \\
m_{\text {ref }}=\frac{\sqrt{I_{\mathrm{d}, \text { ref }}^{2}+I_{\mathrm{q}, \text { ref }}^{2}}}{i_{\mathrm{dc}}}
\end{gathered}
$$

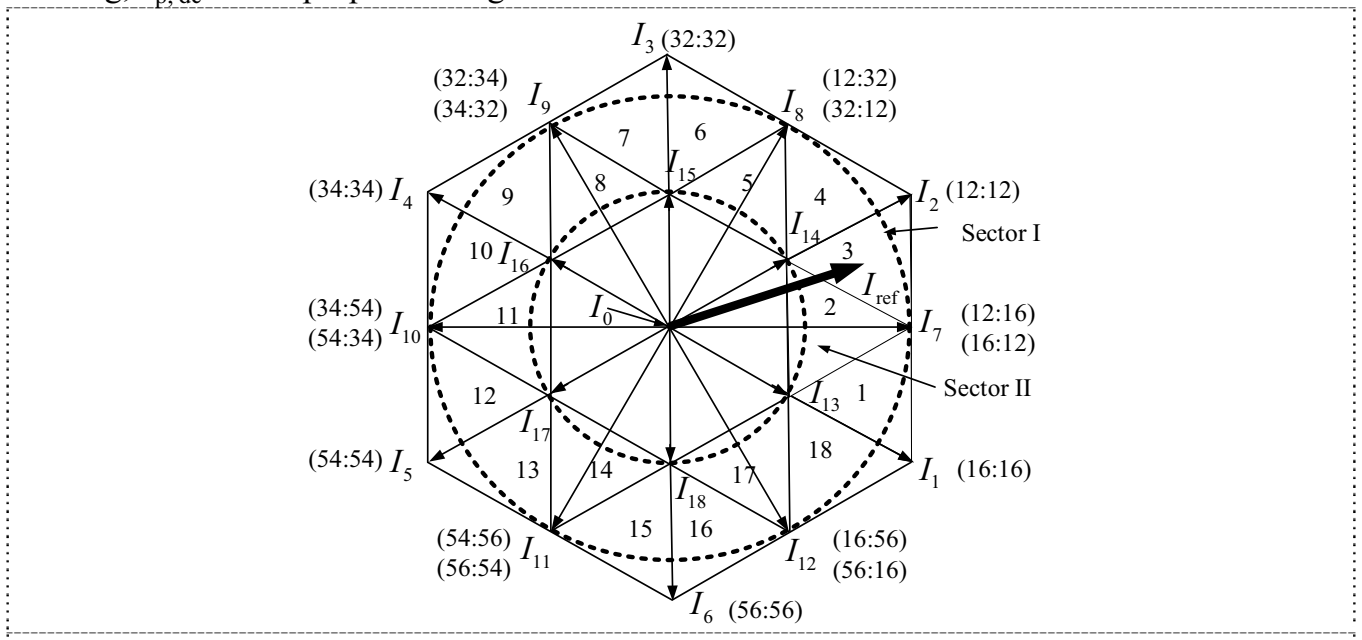

Figure 2. Distribution of current vectors for parallel CSCs.

\subsection{Modulation approach}

For the above-mentioned two modules-based CSCs, to meet with the constrain of CSC, the complete switching table is shown in Table. 1. As seen, the switching states of CSCs are classified into four types, namely large vector, medium vector, small vector and zero vectors, according to the magnitude of the vector as defined in (7). In the table, the number of array stands for the switch number being turned. For instance, the medium vector $(16,12)$ means the switches $S_{1}$ and $S_{6}$ of the CSC module\#1 and switches $S_{1}$ and $S_{2}$ of the CSC module\#2 are turned on. The vectors in Fig. 2 can be further classified into four type based on the magnitude of vectors as: large vectors $I_{1} \sim I_{6}$, medium vectors $I_{7} \sim I_{12}$, small vectors $I_{13} \sim I_{18}$ and zero vectors $I_{0}$.

Table 1. Switching table of parallel CSCs.

(a) Switching state of large vectors SWITCHING STATES

\begin{tabular}{ll}
\hline$I_{1}$ & $16: 16$ \\
$I_{2}$ & $12: 12$
\end{tabular}




\begin{tabular}{|c|c|}
\hline$I_{3}$ & $32: 32$ \\
\hline$I_{4}$ & $34: 34$ \\
\hline$I_{5}$ & $54: 54$ \\
\hline$I_{6}$ & $56: 56$ \\
\hline$I_{7}$ & $56: 56$ \\
\hline$I_{8}$ & $16: 12 \quad 12: 16$ \\
\hline$I_{9}$ & $12: 32 \quad 32: 12$ \\
\hline$I_{10}$ & $32: 34 \quad 34: 32$ \\
\hline$I_{11}$ & $34: 54 \quad 54: 34$ \\
\hline$I_{12}$ & $54: 5656: 54$ \\
\hline & b) Switching state of medium vectors \\
\hline \multicolumn{2}{|r|}{ SWITCHING STATES } \\
\hline$I_{7}$ & $16: 12 \quad 12: 16$ \\
\hline$I_{8}$ & $12: 32 \quad 32: 12$ \\
\hline 19 & $32: 34 \quad 34: 32$ \\
\hline$I_{10}$ & $34: 5454: 34$ \\
\hline$I_{11}$ & $54: 5656: 54$ \\
\hline$I_{12}$ & $16: 5656: 16$ \\
\hline
\end{tabular}

(c) Switching state of small vectors SWITCHING STATES

\begin{tabular}{|c|c|}
\hline$I_{13}$ & 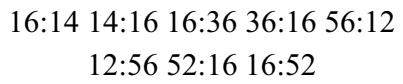 \\
\hline \multirow{2}{*}{$I_{14}$} & $12: 14$ 14:12 12:52 52:12 \\
\hline & $36: 12 \quad 12: 36 \quad 32: 16 \quad 16: 32$ \\
\hline \multirow{2}{*}{$I_{15}$} & $32: 36$ 36:32 32:52 52:32 \\
\hline & $34: 12 \quad 12: 34 \quad 32: 14 \quad 14: 32$ \\
\hline \multirow{2}{*}{$I_{16}$} & $34: 36$ 36:34 14:34 34:14 \\
\hline & $54: 32 \quad 32: 54 \quad 52: 34 \quad 34: 52$ \\
\hline \multirow{2}{*}{$I_{17}$} & $54: 14$ 14:54 54:52 52:54 \\
\hline & $54: 36 \quad 36: 54$ 56:34 34:56 \\
\hline \multirow{2}{*}{$I_{18}$} & $56: 52$ 52:56 56:36 36:56 \\
\hline & $54: 16$ 16:54 56:14 14:56 \\
\hline
\end{tabular}

Table 2. Impact of $I_{12}$ to dc current balancing

\begin{tabular}{llcccc}
\cline { 1 - 1 }$I_{12}$ & & $i_{\mathrm{pc} 1}$ & $i_{\mathrm{nc} 1}$ & $i_{\mathrm{pc} 2}$ & $i_{\mathrm{nc} 2}$ \\
$(16: 56)$ & $u_{\mathrm{sa}}>u_{\mathrm{sc}}$ & $\uparrow$ & $\mathrm{X}$ & $\downarrow$ & $\mathrm{X}$ \\
& $u_{\mathrm{sa}}<u_{\mathrm{sc}}$ & $\downarrow$ & $\mathrm{X}$ & $\uparrow$ & $\mathrm{X}$ \\
$(56: 16)$ & $u_{\mathrm{sa}}>u_{\mathrm{sc}}$ & $\downarrow$ & $\mathrm{X}$ & $\uparrow$ & $\mathrm{X}$ \\
& $u_{\mathrm{sa}}<u_{\mathrm{sc}}$ & $\uparrow$ & $\mathrm{x}$ & $\downarrow$ & $\mathrm{x}$ \\
\hline
\end{tabular}

Table 3. Impact of $I_{13}$ to de current balancing

\begin{tabular}{|c|c|c|c|c|c|c|}
\hline$I_{13}$ & & & $i_{\mathrm{pcl} 1}$ & $i_{\mathrm{nc} 1}$ & $i_{\mathrm{pc} 2}$ & $i_{\mathrm{nc} 2}$ \\
\hline \multirow{4}{*}{$(56: 12)$} & $u_{\mathrm{sa}}>u_{\mathrm{sc}}$ & $u_{\mathrm{sb}}>u_{\mathrm{sc}}$ & $\downarrow$ & $\downarrow$ & $\uparrow$ & $\uparrow$ \\
\hline & $u_{\mathrm{sa}}>u_{\mathrm{sc}}$ & $u_{\mathrm{sb}}<u_{\mathrm{sc}}$ & $\downarrow$ & $\uparrow$ & $\uparrow$ & $\downarrow$ \\
\hline & $u_{\mathrm{sa}}<u_{\mathrm{sc}}$ & $u_{\mathrm{sb}}>u_{\mathrm{sc}}$ & $\uparrow$ & $\downarrow$ & $\downarrow$ & $\uparrow$ \\
\hline & $u_{\mathrm{sa}}<u_{\mathrm{sc}}$ & $u_{\mathrm{sb}}<u_{\mathrm{sc}}$ & $\uparrow$ & $\uparrow$ & $\downarrow$ & $\downarrow$ \\
\hline \multirow{4}{*}{$(12: 56)$} & $u_{\mathrm{sa}}>u_{\mathrm{sc}}$ & $u_{\mathrm{sb}}>u_{\mathrm{sc}}$ & $\uparrow$ & $\uparrow$ & $\downarrow$ & $\downarrow$ \\
\hline & $u_{\mathrm{sa}}>u_{\mathrm{sc}}$ & $u_{\mathrm{sb}}<u_{\mathrm{sc}}$ & $\uparrow$ & $\downarrow$ & $\downarrow$ & $\uparrow$ \\
\hline & $u_{\mathrm{sa}}<u_{\mathrm{sc}}$ & $u_{\mathrm{sb}}>u_{\mathrm{sc}}$ & $\downarrow$ & $\uparrow$ & $\uparrow$ & $\downarrow$ \\
\hline & $u_{\mathrm{sa}}<u_{\mathrm{sc}}$ & $u_{\mathrm{sb}}<u_{\mathrm{sc}}$ & $\downarrow$ & $\downarrow$ & $\uparrow$ & $\uparrow$ \\
\hline \multirow{4}{*}{$(52: 16)$} & $u_{\mathrm{sa}}>u_{\mathrm{sc}}$ & $u_{\mathrm{sb}}>u_{\mathrm{sc}}$ & $\downarrow$ & $\uparrow$ & $\uparrow$ & $\downarrow$ \\
\hline & $u_{\mathrm{sa}}>u_{\mathrm{sc}}$ & $u_{\mathrm{sb}}<u_{\mathrm{sc}}$ & $\downarrow$ & $\downarrow$ & $\uparrow$ & $\uparrow$ \\
\hline & $u_{\mathrm{sa}}<u_{\mathrm{sc}}$ & $u_{\mathrm{sb}}>u_{\mathrm{sc}}$ & $\uparrow$ & $\uparrow$ & $\downarrow$ & $\downarrow$ \\
\hline & $u_{\mathrm{sa}}<u_{\mathrm{sc}}$ & $u_{\mathrm{sb}}<u_{\mathrm{sc}}$ & $\uparrow$ & $\downarrow$ & $\downarrow$ & $\uparrow$ \\
\hline \multirow{4}{*}{$(16: 52)$} & $u_{\mathrm{sa}}>u_{\mathrm{sc}}$ & $u_{\mathrm{sb}}>u_{\mathrm{sc}}$ & $\uparrow$ & $\downarrow$ & $\downarrow$ & $\uparrow$ \\
\hline & $u_{\mathrm{sa}}>u_{\mathrm{sc}}$ & $u_{\mathrm{sb}}<u_{\mathrm{sc}}$ & $\uparrow$ & $\uparrow$ & $\downarrow$ & $\downarrow$ \\
\hline & $u_{\mathrm{sa}}<u_{\mathrm{sc}}$ & $u_{\mathrm{sb}}>u_{\mathrm{sc}}$ & $\downarrow$ & $\downarrow$ & $\uparrow$ & $\uparrow$ \\
\hline & $u_{\mathrm{sa}}<u_{\mathrm{sc}}$ & $u_{\mathrm{sb}}<u_{\mathrm{sc}}$ & $\downarrow$ & $\uparrow$ & $\uparrow$ & $\downarrow$ \\
\hline
\end{tabular}

The selection of vectors and their dwell time shall be determined according to the "current-ampere" balance principle and the balancing of dc rail current.

It has been discussed in [12] that the small vectors are most effective for dc current sharing. In addition, considering the de-icing equipment often operate in a region with varying modulation index, it is also necessary to use the medium vector for dc current balancing. For instance, when the medium vector $I_{12}$ is chosen, there are two redundant switching sates (16:56) and $(56: 16)$ can be selected. In this case, the dc current balancing is actually determined by the dc terminal voltage of each module. It is also necessary to note that the dc terminal voltage is determined by the switching state of CSC modules and the instantaneous value of grid phase voltage. Considering different rankings of instantaneous grid phase voltage values, it is easy to obtain Table 2, where both the impact of switching state (56:16) and (16:56) under various grid voltage value rankings are listed. Further investigation of the table finds that when the vector $I_{12}$ is selected, it can always find suitable switching state to reduce the dc current balancing errors. Similarly, the performance of small vectors to dc current balancing is also listed in Table 3 . During the operation of the de-icing equipment, the dynamic dc current balancing is achieved by the joint impact of both small and medium vectors.

Finally, the complete diagram of the proposed parallel CSC module based de-icing system is shown in Fig. 3, where the simplified power circuit is shown in the upper part and the control scheme is shown in the lower part. 


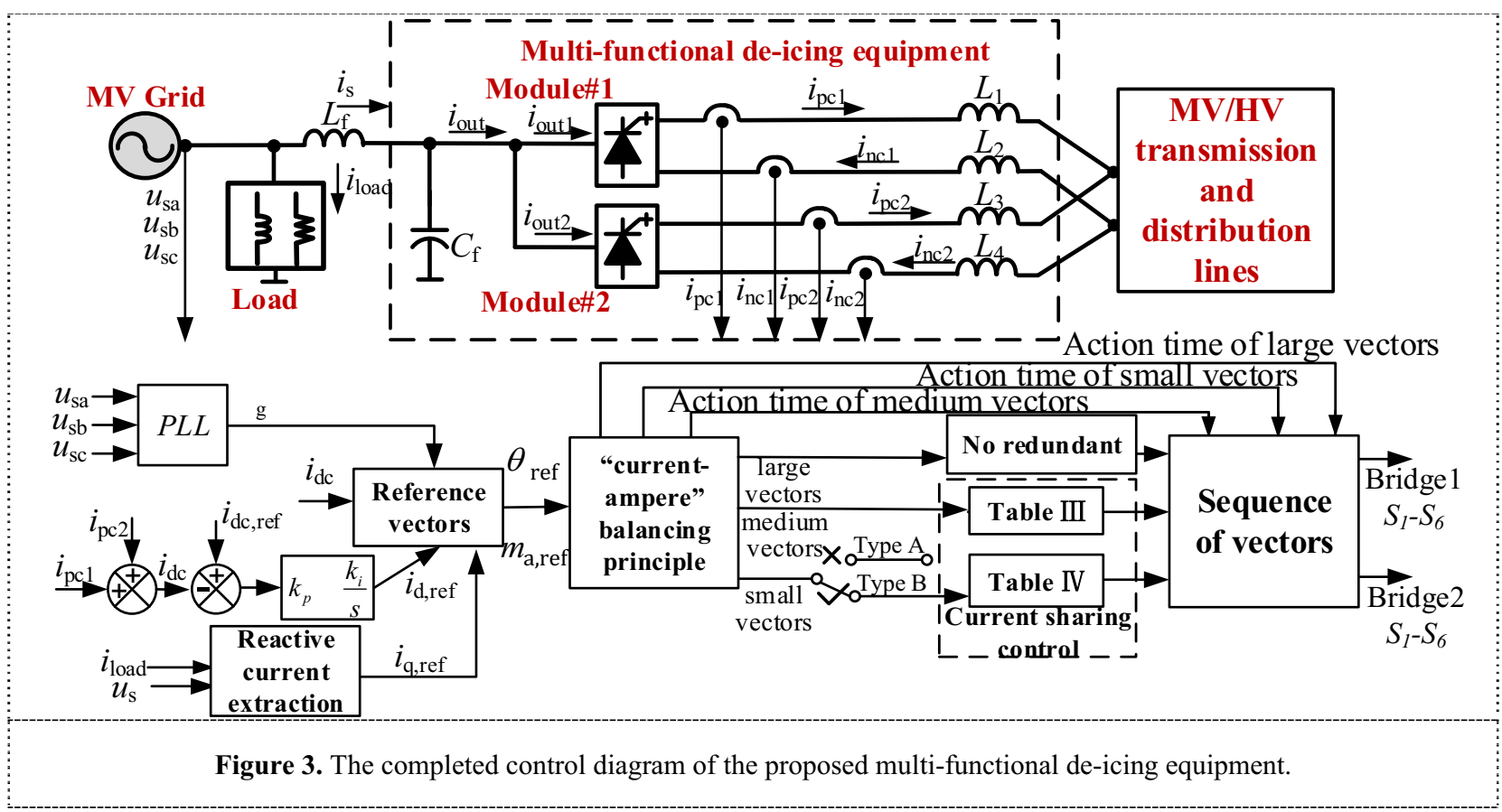

As seen, the controller has a phase-locked loop (PLL) to obtain the instantaneous grid voltage phase angle $\theta_{\mathrm{g}}$. The measured total dc current $i_{\mathrm{dc}}$ is compared with the reference current $i_{\mathrm{dc} \text {, ref }}$ and a PI regulation is used to get the d-axis reference ac current as $i_{\mathrm{d} \text {, ref. }}$ In addition to that, the load current $i_{\text {load }}$ is also measured by the controller and its reactive power component $i_{\mathrm{q} \text {, ref }}$ is extracted and it is adopted as q-axis component of the reference current. Afterwards, the angle of reference current $\theta_{\text {ref }}$ and the modulation index is determined.

Then, according to the "current-ampere" balancing principle, the dwell time and type of small and medium vectors are both determined. Finally, the dc currents of each dc rail are collected, and the switching states of small and medium vectors are determined according to the distribution of dc rail current, according to Table 2 and Table 3.

Table 4. Circuit and control parameters.

\begin{tabular}{lc}
\hline Parameters & VALUE \\
\hline Grid voltage(line to line $)$ & $4160 \mathrm{~V} / 60 \mathrm{~Hz}$ \\
Filter capacitance $\left(C_{\mathrm{f}}\right)$ & $50 \mathrm{uF}$ \\
Filter inductance $\left(L_{\mathrm{f}}\right)$ & $3 \mathrm{mH}$ \\
LV load resistance $\left(R_{\text {load,LV}}\right)$ & $5 \Omega$ \\
LV load inductance $\left(L_{\text {load,LV }}\right)$ & $15 \mathrm{mH}$ \\
DC link inductance $\left(L_{\mathrm{dc}}\right)$ & $52 \mathrm{mH}$ \\
$k_{\mathrm{p}}$ & 0.1 \\
$k_{i}$ & 50 \\
\hline
\end{tabular}

\section{Verification results}

In order to validate the effectiveness of the proposed CSC based de-icing equipment, relevant simulation has been carried out by SIMULINK. The key circuit and control parameters of the system can be seen in Table 4 .

\subsection{Conventional approach}

Firstly, the performance of the system using conventional approach is obtained. Fig. 4 shows the three-phase grid voltage and grid current under the case of conventional control. It is seen that that the grid current phase angle is clearly lagging of grid voltage, due to the presence of a RL load at PCC. 


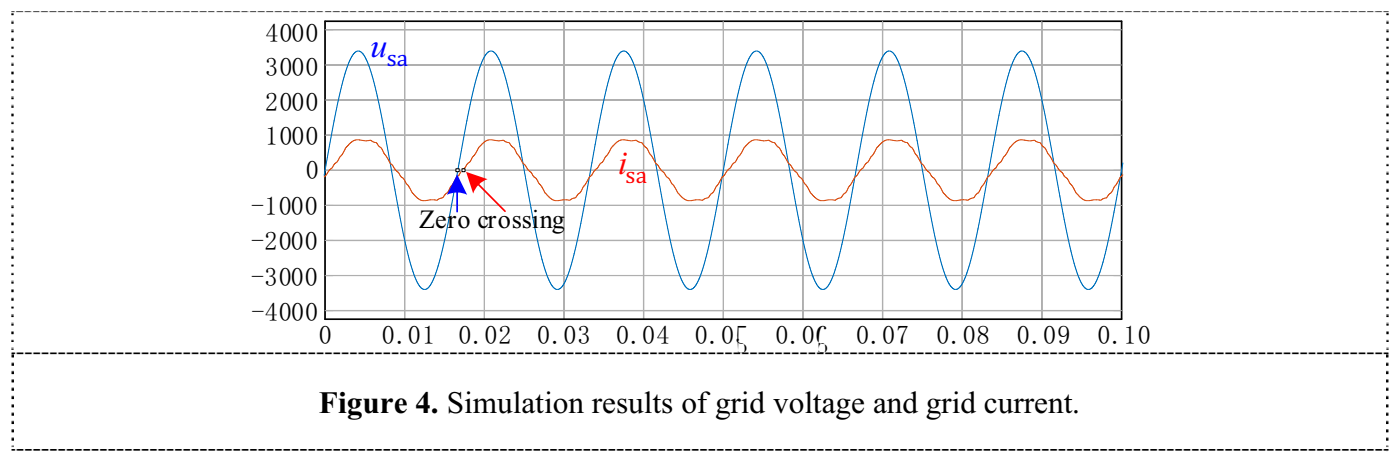

In addition, Fig. 5 shows the three-phase line current of the de-icing equipment and the output current of each CSC module. As seen, the line current of the de-icing equipment is in phase with the grid voltage. However, the output current magnitudes of parallel CSC modules are clearly not the same, due to a fact that conventional control method does not have an active control on the dc rail current balancing.

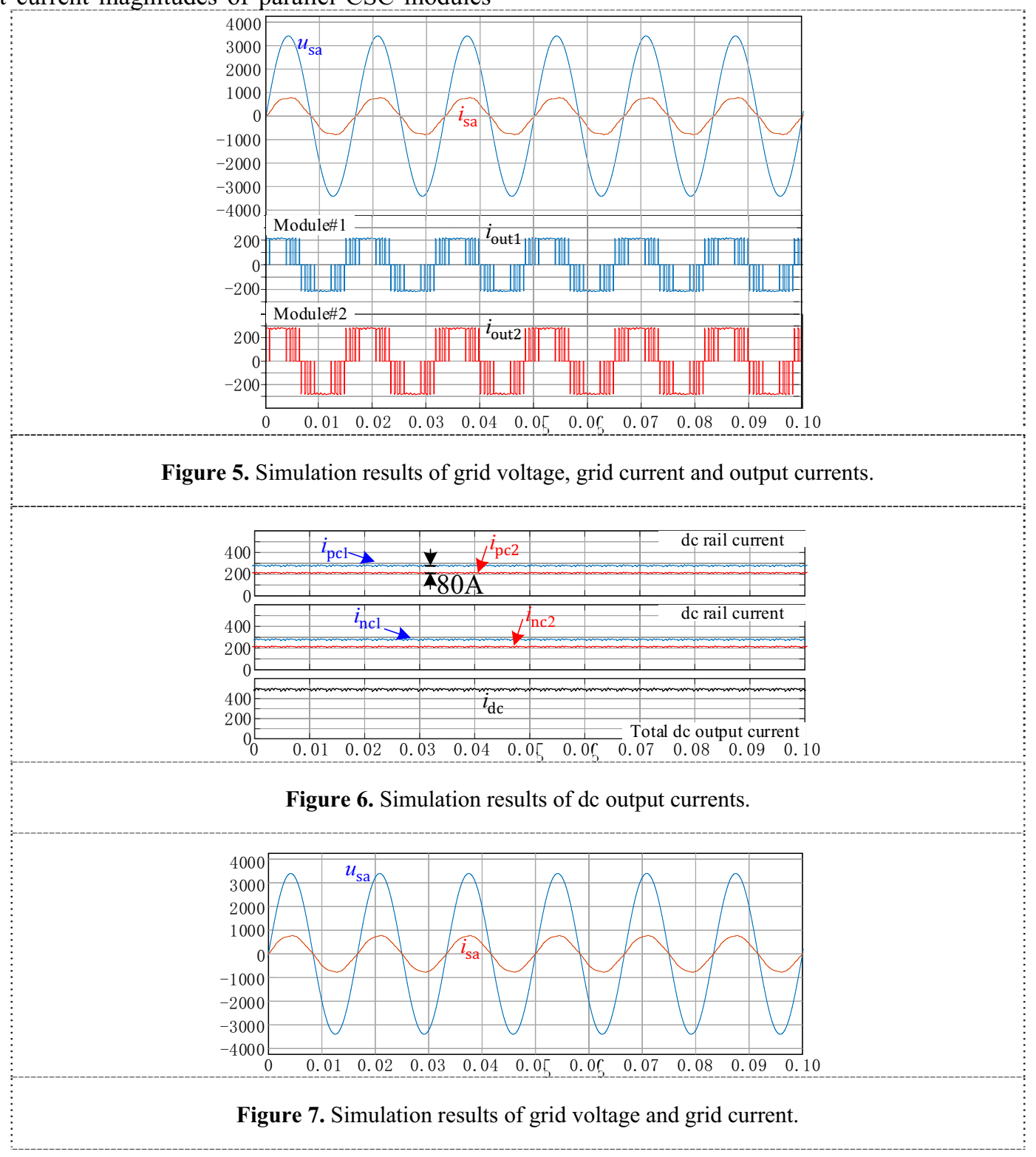




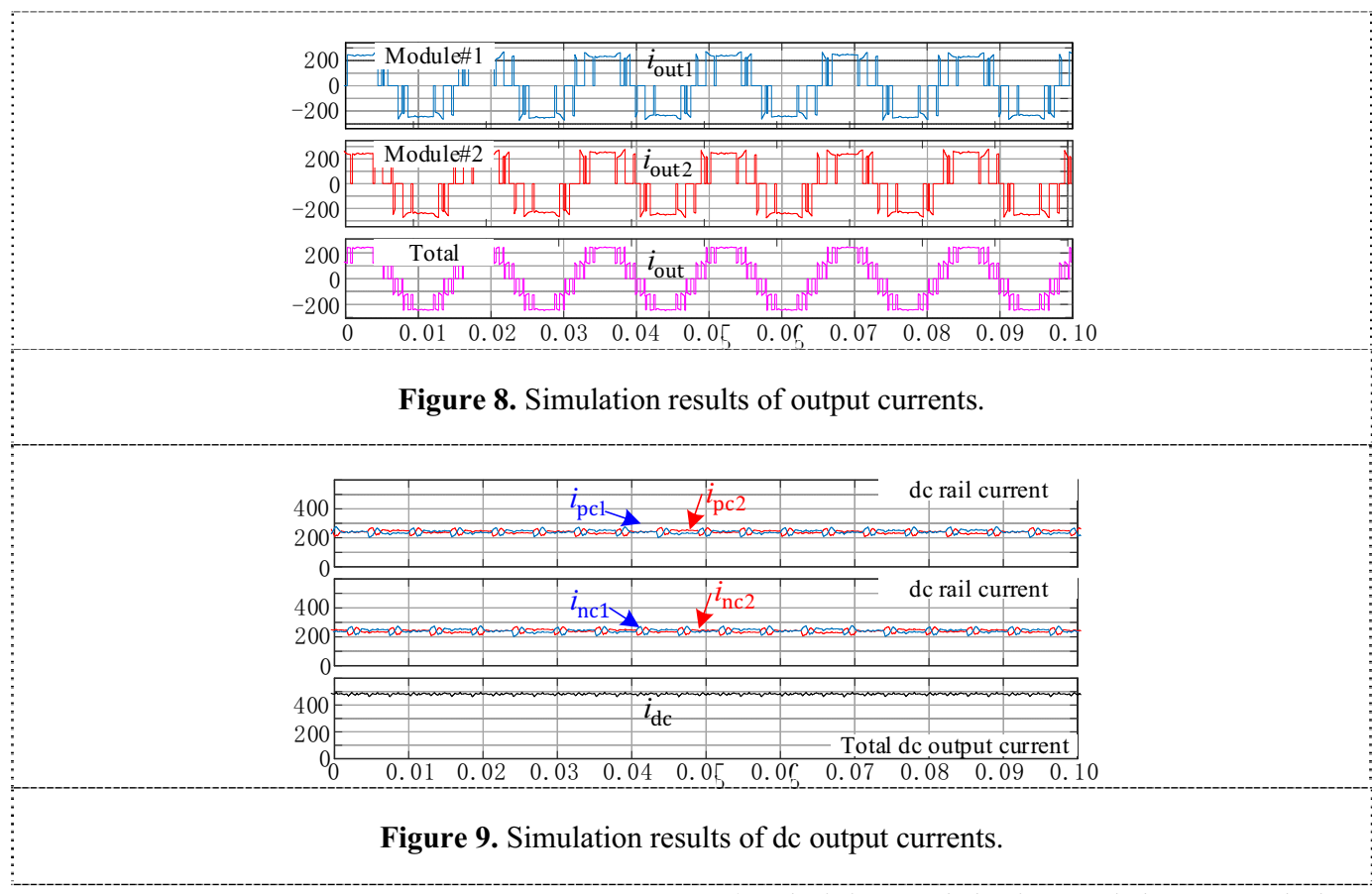

The detailed performance of total dc output current and de rail currents is shown in Fig. 6. In the case of using conventional control, it can be seen that the total output current can reach the reference value at $500 \mathrm{~A}$ at the steady-state. However, the output current is not equally distributed on the CSC module dc rail. The difference between CSC module dc rail current can be up to $80 \mathrm{~A}$. This clearly bring different stress on the CSC modules.

\subsection{The proposed approach}

Similar simulation is conducted by using the proposed method and corresponding results are shown from Fig. 7 to Fig. 9 .

Firstly, the three-phase grid voltage and grid current of the system are shown in Fig. 7. In this case, due to the active compensation of local load reactive power by using CSCs, it can be seen that the grid current and grid voltage are almost in phase.

In addition, the de-icing equipment line current and the output current of each CSC module are shown in Fig. 8. As the dc rail current is well balanced by using the proposed approach, it is seen that the output current of both CSC has the rough the same magnitude at around 250A.

Finally, the total dc output current performance and the dc rail current sharing performance are shown in Fig. 9. As expected, the control of total dc output current is accurate at 500A. In addition, both the upper dc rail and lower dc rail of CSCs modules have a good equal sharing of total dc current.

\section{Conclusions}

The paper proposed a multi-functional CSC based icing equipment. The proposed system consists of parallel CSC modules to enhance the dc current output capacity for de-icing and the internal dc current balancing among parallel CSC modules is achieved by online selecting the switching state of medium and small vectors. In addition, the proposed system can easily realize proper low voltage distribution network reactive power compensation in a direct current modulation manner to ensure unity power factor operation. Experimental results have been provided to validate the effectiveness of the proposed approach.

\section{References}

1. Koval and A.A.Chowdhury, "An investigation into extremeweather caused transmission line unavailability," in Proc. IEEE Power Eng. Soc. Gen. Meeting, 2005, pp.2425-2428.

2. M.Farzaneh,"Systems for prediction and monitoring of iceshedding, anti-icing and de-icing for overhead lines," CIGRÉ Working Group B2.29, Electrano. 25, pp.51-573, Dec. 2010.

3. J. Lu, M. Zeng, X. Zeng, Z. Fang and J. Yuan, "Analysis of ice-covering characteristics of China Hunan power grid", IEEE Trans. Ind. Appl., vol. 51, no. 3, pp. 1997-2002, May/Jun. 2015.

4. C. Wang, J. Wen, S. Li, X. Ma and J. Wang, "Design on DC de-icing schemes for high voltage transmission line", Proc. 5th Critical Infrastructure Int. Conf., pp. 1-5, Sep. 2010.

5. S. Bhattacharya, Z. Xi, B. Fardenesh and E. Uzunovic, "Control reconfiguration of VSC based STATCOM for de-icer application", Proc. IEEE Power Eng. Soc. Gen. Meeting, pp. 1-7, 2008.

6. L. Ding, Z. Quan and Y. W. Li, "Common-mode voltage reduction for parallel CSC-fed motor drives with multilevel modulation", IEEE Trans. Power Electron., vol. 33, no. 8, pp. 6555-6566, Aug. 2018. 
7. X. Guo, N. Wang, J. Zhang, B. Wang and M. Nguyen, "A novel transformerless current source inverter for leakage current reduction", IEEE Access, vol. 7, pp. 50681-50690, 2019.

8. L. Ding, Y. Lian and Y. W. Li, "Multilevel current source converters for high power medium voltage applications", CES Trans. Elect. Mach. Syst., vol. 1, no. 3, pp. 306-314, Sep. 2017.

9. H. Akagi, "New trends in active power filters for power conditioning," IEEE Trans. Ind. Applicat., vol. 32, no. 6, pp. 1312-1322, Nov/Dec. 1996.

10. M. Triggianese, F. Liccardo, and P. Marino, "Ancillary services performed by distributed generation in grid integration," in Proc. IEEE Int. Conf. Clean Elect. Power, 2007, pp. 164-170.

11. C. J. Gajanayake, D. M. Vilathgamuwa, P. C. Loh, R. Teodorescu, and F. Blaabjerg, "Z-sourceinverter-based flexible distributed generation system solution for grid power quality improvement," IEEE Trans. Energy Conversion, vol.24, pp.695-704, Sep. 2009.

12. R. I. Bojoi, L. R. Limongi, D. Roiu, and A. Tenconi, "Enhanced power quality control strategy for single-phase inverters in distributed generation systems," IEEE Trans. Power Electron., vol. 26, no.3, pp. 798-806, Mar. 2011. 